\title{
Deformed Double Yangian Structures
}

\author{
D. Arnaudon ${ }^{a}$, J. Avan ${ }^{b}$, L. Frappat ${ }^{a}$, M. Rossi ${ }^{c}$ \\ ${ }^{a}$ Laboratoire d'Annecy-le-Vieux de Physique Théorique LAPTH \\ CNRS, URA 1436, associée à l'Université de Savoie \\ LAPP, BP 110, F-74941 Annecy-le-Vieux Cedex, France \\ ${ }^{b}$ LPTHE, CNRS, UMR 7589, Universités Paris VI/VII, France \\ ${ }^{c}$ Department of Mathematics, University of Durham \\ South Road, Durham DH1 3LE, UK
}

\begin{abstract}
Scaling limits at $q \rightarrow 1$ of the elliptic vertex algebras $\mathcal{A}_{q, p}\left(\widehat{s l}(N)_{c}\right)$ are defined for any $N$, extending the previously known case of $N=2$. They realise deformed, centrally extended double Yangian structures $\mathcal{D} Y_{r}(s l(N))_{c}$. As in the quantum affine algebras $\mathcal{U}_{q}\left(\widehat{s l}(N)_{c}\right)$, and quantum elliptic affine algebras $\mathcal{A}_{q, p}\left(\widehat{s l}(N)_{c}\right)$, these algebras contain subalgebras at critical values of the central charge $c=-N-M r(M$ integer, $2 r=\ln p / \ln q)$, which become Abelian when $c=-N$ or $2 r=N h$ for $h$ integer. Poisson structures and quantum exchange relations are derived for their abstract generators.
\end{abstract}

MSC number: 81R50, 17B37

LAPTH-716/99

PAR-LPTHE 99-05

DTP-99-7

math.QA/9905100

February 1999

Revised: May 1999 


\section{Introduction}

Elliptic deformation of a finite Lie algebra was first proposed by Sklyanin [1]. The incorporation of a central extension leading to the notion of vertex elliptic affine algebra $\mathcal{A}_{q, p}\left(\widehat{s l}(2)_{c}\right)$ was achieved in [2] in the $R$-matrix formalism using Belavin-Baxter 8-vertex matrix [3]. Its quasi-Hopf structure as a twist of $\mathcal{U}_{q}\left(\widehat{s l}(2)_{c}\right)$ was elucidated in [⿶]; a universal formula for the twist was proposed in [5] and allowed to define an $R$-matrix formulation for the general case $\mathcal{A}_{q, p}\left(\widehat{s l}(N)_{c}\right)$.

Vertex elliptic affine algebras were shown to possess at least two inequivalent limits with one parameter less. The limit $p \rightarrow 0$, together with a suitable renormalisation of the generators, leads back to the $R$-matrix structure of the quantum group $\mathcal{U}_{q}\left(\widehat{s l}(N)_{c}\right)$ as shown in [2] for $N=2$.

Another limit was established for $N=2$ in [6], defining a so-called scaled limit $\mathcal{A}_{\hbar, \eta}\left(\widehat{s l}(2)_{c}\right)$. It was also described in [7, 8]. It is obtained by sending $p, q$ and the spectral parameter $z$ to 1 while retaining as finite parameters $\ln p / \ln q$ and $\ln z / \ln q$. A subsequent $\operatorname{limit} \ln p / \ln q \rightarrow \infty$ leads [6] to an algebraic structure of same $R$-matrix formulation as the centrally extended double Yangian $\mathcal{D} Y(s l(2))_{c}$ introduced in [9, 10]. Other definitions using differently normalised $R$-matrices have been proposed for this object [11, 12]. The scaled algebra $\mathcal{A}_{\hbar, \eta}\left(\widehat{s l}(2)_{c}\right)$ was recently shown [13] to be realised by the screening currents for the Sine-Gordon model at the free fermion point (see also [17, 8]). At this time, only for $N=2$ has the scaling limit been defined.

It has been known for a while that quantum affine algebras $\mathcal{U}_{q}(\widehat{\mathcal{G}})_{c}$ admit infinite dimensional centres at critical values of the central charge [14]. Associated non-linear Poisson structures realising $q$-deformed classical $\mathcal{W}_{N}$ algebras were derived for $\mathcal{G}=\operatorname{sl}(N)$ [15, 16] and quantised [16, [17]. A similar, albeit more complex, structure of sub-exchange algebras, extended centres or Abelian algebras, and classical (Poisson bracket) limits thereof, was uncovered for $\mathcal{A}_{q, p}\left(\widehat{s l}(2)_{c}\right)$ 18, 19] and $\mathcal{A}_{q, p}\left(\widehat{s l}(N)_{c}\right)[20,21]$. They realise new quantum versions of the already known $q-\mathcal{W}_{N}$ Poisson structure [15, 16]. No such results are known for the scaled algebras $\mathcal{A}_{\hbar, \eta}\left(\widehat{s l}(2)_{c}\right)$ ] and their yet to be defined generalisations to $\operatorname{sl}(N)$.

Our purpose therefore is to apply to the scaled limits $\mathcal{A}_{\hbar, \eta}\left(\widehat{s l}(N)_{c}\right)$, once they are consistently defined, the same systematic study undertaken in [15] for affine quantum algebras and [18, 19, 20, 21] for vertex elliptic affine algebras, i.e. deduce from the $R$-matrix formulation the existence of extended centres (or at least Abelian subalgebras), and compute the related Poisson structures and the related quantisations.

The ultimate purpose is to try to define new types of classical and quantum deformed $\mathcal{W}_{N^{-}}$ algebras. However a particular difficulty arises here owing to the current state of understanding of scaled algebras as univocally defined algebraic structures. Indeed, definition of the full algebraic structure requires a proper definition of the mode expansion both for generating functionals and structure functions, and there may exist several inequivalent expansions (and therefore inequivalent algebras) for one single abstract structure. The structure functions here turn out to be single-period meromorphic functions with an essential singularity at infinity. It was commented in [6] that (in the rational limit) two different structures could be defined from one single $R$-matrix formulation, depending whether the modes of the generators were defined to be continuous-labelled Fourier transforms with respect to the spectral parameter $\beta$, leading to the algebra $\mathcal{A}_{\hbar, 0}\left(\widehat{s l}(2)_{c}\right)$ [6], or discrete-labelled coefficient of power expansion in $\beta$, leading to the double Yangian $\mathcal{D} Y(\operatorname{sl}(2))_{c}$ [9, 10]. Similarly the deformed version of the exchange algebra, and the subsequent Poisson structures on the critical surfaces, will give rise to inequivalent algebraic structures depending upon the type of mode expansion

\footnotetext{
${ }^{1}$ This was pointed out to us by V. Korepin and P. Sorba.
} 
eventually chosen. This discussion goes beyond the scope of the current presentation and will be left for further studies. Identification of, and discussions on algebraic structures are thus generically understood here at the level of the generating functionals and $R$ matrix structures.

Within this framework we address the two basic problems of this paper as follows:

In a first part, we define an $R$-matrix formulation for a scaling limit $q \rightarrow 1$ of the vertex algebra $\mathcal{A}_{q, p}\left(\widehat{s l}(N)_{c}\right)$. We prove explicitly that the scaling limit structure matrix obeys the Yang-Baxter equation, unitarity, crossing-symmetry and quasi-periodicity relations. We define a subsequent limit $\ln p / \ln q \rightarrow \infty$. Its $R$-matrix structure is strongly reminiscent of the double Yangian $\mathcal{D} Y(s l(N))_{c}$ in the formulation of [11, 12], albeit with a different normalisation factor. As a consequence the structure at $2 r=\ln p / \ln q<\infty$ can be interpreted as a deformation of the double Yangian of which more will be said in the conclusion, hence it will be denoted $\mathcal{D} Y_{r}(\operatorname{sl}(N))_{c}$.

In a second part, we show that the scaled algebra $\mathcal{D} Y_{r}(\operatorname{sl}(N))_{c}$ contains non-linear exchange subalgebras when $c=-N-M r$ for $M \in \mathbb{Z}$. When $M=0$ (that is $c=-N$ ) these algebras are Abelian. When $M \neq 0$, the algebras become Abelian when $2 r=N h=2 r_{\text {crit }}$ for $h \in \mathbb{Z} \backslash\{0\}$. Non-linear Poisson structures are then derived on these Abelian subalgebras, and the exchange algebras at $M \neq 0$ can be interpreted as consistent quantisations inside $\mathcal{D} Y_{r}(s l(N))_{c}$ of the Poisson structures with $\hbar \sim 2\left(r-r_{\text {crit }}\right)$. They contain higher-spin operators obtained as before 21] by the $R$-matrix "twisted" trace formula of the Lax operators. It appears here that the existence of exchange subalgebras on critical surfaces and Abelian limits thereof on critical subalgebras, survive the scaling limit simply by taking directly the same scaling limit in the characteristic relations and the structure functions. Interpretations of the Poisson algebras and exchange algebras, in connection with Virasoro and $\mathcal{W}_{N}$ algebras, require to establish the exact mode expansion to be used on these generating functionals, and will be left for further studies.

In conclusion we indicate several directions of interest which we mean to pursue in the next future.

\section{Degeneration limit of the elliptic algebra $\mathcal{A}_{q, p}\left(\widehat{s l}(N)_{c}\right)$}

\subsection{Definition of the $\operatorname{sl}(N)$ elliptic $R$-matrix}

The $\operatorname{sl}(N)$ elliptic $R$-matrix in $\operatorname{End}\left(\mathbb{C}^{N}\right) \otimes \operatorname{End}\left(\mathbb{C}^{N}\right)$, associated to the $\mathbb{Z}_{N}$-vertex model, is defined as follows [22, 23]:

$$
R(z, q, p)=z^{2 / N-2} \frac{1}{\kappa\left(z^{2}\right)} \frac{\vartheta\left[\begin{array}{c}
\frac{1}{2} \\
\frac{1}{2}
\end{array}\right](\zeta, \tau)}{\vartheta\left[\begin{array}{c}
\frac{1}{2} \\
\frac{1}{2}
\end{array}\right](\xi+\zeta, \tau)} \sum_{\left(\alpha_{1}, \alpha_{2}\right) \in \mathbb{Z}_{N} \times \mathbb{Z}_{N}} W_{\left(\alpha_{1}, \alpha_{2}\right)}(\xi, \zeta, \tau) I_{\left(\alpha_{1}, \alpha_{2}\right)} \otimes I_{\left(\alpha_{1}, \alpha_{2}\right)}^{-1},
$$

where the variables $z, q, p$ are related to the variables $\xi, \zeta, \tau$ by

$$
z=e^{i \pi \xi}, \quad q=e^{i \pi \zeta}, \quad p=e^{2 i \pi \tau} .
$$

The Jacobi theta functions with rational characteristics $\gamma=\left(\gamma_{1}, \gamma_{2}\right) \in \frac{1}{N} \mathbb{Z} \times \frac{1}{N} \mathbb{Z}$ are defined by:

$$
\vartheta\left[\begin{array}{l}
\gamma_{1} \\
\gamma_{2}
\end{array}\right](\xi, \tau)=\sum_{m \in \mathbb{Z}} \exp \left(i \pi\left(m+\gamma_{1}\right)^{2} \tau+2 i \pi\left(m+\gamma_{1}\right)\left(\xi+\gamma_{2}\right)\right) .
$$

The normalisation factor is chosen as follows:

$$
\frac{1}{\kappa\left(z^{2}\right)}=\frac{\left(q^{2 N} z^{-2} ; p, q^{2 N}\right)_{\infty}\left(q^{2} z^{2} ; p, q^{2 N}\right)_{\infty}\left(p z^{-2} ; p, q^{2 N}\right)_{\infty}\left(p q^{2 N-2} z^{2} ; p, q^{2 N}\right)_{\infty}}{\left(q^{2 N} z^{2} ; p, q^{2 N}\right)_{\infty}\left(q^{2} z^{-2} ; p, q^{2 N}\right)_{\infty}\left(p z^{2} ; p, q^{2 N}\right)_{\infty}\left(p q^{2 N-2} z^{-2} ; p, q^{2 N}\right)_{\infty}}
$$


The functions $W_{\left(\alpha_{1}, \alpha_{2}\right)}$ are given by

$$
W_{\left(\alpha_{1}, \alpha_{2}\right)}(\xi, \zeta, \tau)=\frac{\vartheta\left[\begin{array}{c}
\frac{1}{2}+\alpha_{1} / N \\
\frac{1}{2}+\alpha_{2} / N
\end{array}\right](\xi+\zeta / N, \tau)}{N \vartheta\left[\begin{array}{c}
\frac{1}{2}+\alpha_{1} / N \\
\frac{1}{2}+\alpha_{2} / N
\end{array}\right](\zeta / N, \tau)} .
$$

The matrices $I_{\left(\alpha_{1}, \alpha_{2}\right)}$ are defined by

$$
I_{\left(\alpha_{1}, \alpha_{2}\right)}=g^{\alpha_{2}} h^{\alpha_{1}}
$$

where the $N \times N$ matrices $g$ and $h$ are given by $g_{i j}=\omega^{i} \delta_{i j}$ and $h_{i j}=\delta_{i+1, j}$, the addition of indices being understood modulo $N$ and $\omega=e^{2 i \pi / N}$.

Let us set

$$
S(\xi, \zeta, \tau)=\sum_{\left(\alpha_{1}, \alpha_{2}\right) \in \mathbb{Z}_{N} \times \mathbb{Z}_{N}} W_{\left(\alpha_{1}, \alpha_{2}\right)}(\xi, \zeta, \tau) I_{\left(\alpha_{1}, \alpha_{2}\right)} \otimes I_{\left(\alpha_{1}, \alpha_{2}\right)}^{-1} .
$$

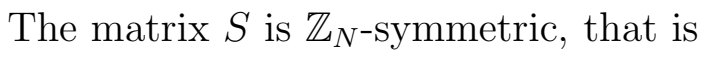

$$
S_{a+s, b+s}^{c+s, d+s}=S_{a, b}^{c, d}
$$

for any indices $a, b, c, d, s \in \mathbb{Z}_{N}$ (the addition of indices being understood modulo $N$ ) and the nonvanishing elements of the matrix $S$ are of the type $S_{a, b}^{c, a+b-c}$. One finds explicitly:

$$
S_{a, b}^{c, a+b-c}(\xi, \zeta, \tau)=\frac{\vartheta\left[\begin{array}{c}
\left.(b-a) / N+\frac{1}{2}\right] \\
\frac{1}{2}
\end{array}\right](\xi+\zeta, N \tau)}{\vartheta\left[\begin{array}{c}
(c-a) / N+\frac{1}{2} \\
\frac{1}{2}
\end{array}\right](\zeta, N \tau)} \frac{\prod_{k=0, k \neq b-c}^{N-1} \vartheta\left[\begin{array}{c}
k / N+\frac{1}{2} \\
\frac{1}{2}
\end{array}\right](\xi, N \tau)}{\prod_{k=1}^{N-1} \vartheta\left[\begin{array}{c}
k / N+\frac{1}{2} \\
\frac{1}{2}
\end{array}\right](0, N \tau)}
$$

Using the relation

$$
\prod_{k=1}^{N-1} \vartheta\left[\begin{array}{c}
k / N+\frac{1}{2} \\
\frac{1}{2}
\end{array}\right](\xi, N \tau)=p^{(N-1)(N-2) / 24} \frac{\left[\prod_{m=1}^{\infty}\left(1-p^{N m}\right)\right]^{N}}{\prod_{m=1}^{\infty}\left(1-p^{m}\right)} \frac{\Theta_{p}\left(z^{2}\right)}{\Theta_{p^{N}}\left(z^{2}\right)}
$$

one finds the following expression in terms of the Jacobi $\Theta$ functions for the elements $R_{a, b}^{c, a+b-c}$ of the matrix $R(z, q, p)$ :

$$
\begin{aligned}
R_{a, b}^{c, a+b-c}=\frac{1}{\kappa\left(z^{2}\right)} z^{\frac{2}{N}(1-a)} q^{\frac{2 b}{N}} p^{-\frac{(a-c)(b-c)}{N}} \frac{\left[\prod_{m=1}^{\infty}\left(1-p^{N m}\right)\right]^{3}}{\left[\prod_{m=1}^{\infty}\left(1-p^{m}\right)\right]^{3}} \\
\times \frac{\Theta_{p^{N}}\left(p^{N+b-a} q^{2} z^{2}\right)}{\Theta_{p^{N}}\left(p^{N+b-c} z^{2}\right) \Theta_{p^{N}}\left(p^{N-a+c} q^{2}\right)} \frac{\Theta_{p}\left(q^{2}\right) \Theta_{p}\left(p z^{2}\right)}{\Theta_{p}\left(q^{2} z^{2}\right)} .
\end{aligned}
$$




\subsection{Scaling limit of the $\operatorname{sl}(N)$ elliptic $R$-matrix}

The scaling limit of the $R$ matrix (2.11) is defined by

$$
q \rightarrow 1, \quad z=q^{i \beta / \pi}, \quad p=q^{2 r}, \quad \text { where } \beta, r \text { are kept fixed } .
$$

Evaluation of the limit of the elements (2.11) requires regularisation of potentially divergent terms (for instance by exchanging limits and infinite products symbols). This in turn implies that the crucial identities (such as Yang-Baxter equation, unitarity, ...) of the scaling limit of the $R$ matrix be checked again (see Theorem 11).

One finds in the limit (2.12) that

$$
\frac{\Theta_{p^{n}}\left(p^{u} q^{2 v} z^{2 m}\right)}{\Theta_{p^{n^{\prime}}}\left(p^{u^{\prime}} q^{2 v^{\prime}} z^{2 m^{\prime}}\right)} \rightarrow \frac{\sin \frac{\pi}{n r}(u r+v+i m \beta / \pi)}{\sin \frac{\pi}{n^{\prime} r}\left(u^{\prime} r+v^{\prime}+i m^{\prime} \beta / \pi\right)} .
$$

It follows that in the scaling limit the matrix elements (2.11) become

$$
R_{a, b}^{c, a+b-c}(\beta, r)=-\frac{1}{N} S_{0}(\beta) \frac{\sin \frac{i \beta+\pi+(b-a) \pi r}{N r}}{\sin \frac{i \beta+(b-c) \pi r}{N r} \sin \frac{\pi-(a-c) \pi r}{N r}} \frac{\sin \frac{i \beta}{r} \sin \frac{\pi}{r}}{\sin \frac{i \beta+\pi}{r}} .
$$

Remark that the factor $1 / N$ arises from a regularisation of the limit of the ratio of the infinite product over $m$ in (2.11). More precisely, the infinite product arises from the $\xi \rightarrow 0$ limit of a ratio of theta functions. The regularisation requires to exchange the limits $\xi \rightarrow 0$ with the limit $q \rightarrow 1$ in this ratio of theta functions.

The normalisation factor $S_{0}(\beta)$ is defined by

$$
S_{0}(\beta)=\frac{S_{2}\left(-\frac{i \beta}{\pi} \mid r, N\right) S_{2}\left(1+\frac{i \beta}{\pi} \mid r, N\right)}{S_{2}\left(\frac{i \beta}{\pi} \mid r, N\right) S_{2}\left(1-\frac{i \beta}{\pi} \mid r, N\right)},
$$

where $S_{2}\left(x \mid \omega_{1}, \omega_{2}\right)$ is the Barnes' double sine function [24] of periods $\omega_{1}$ and $\omega_{2}$ defined by

$$
S_{2}\left(x \mid \omega_{1}, \omega_{2}\right)=\frac{\Gamma_{2}\left(\omega_{1}+\omega_{2}-x \mid \omega_{1}, \omega_{2}\right)}{\Gamma_{2}\left(x \mid \omega_{1}, \omega_{2}\right)},
$$

where

$$
\Gamma_{2}\left(x \mid \omega_{1}, \omega_{2}\right)=\exp \left(\left.\frac{\partial}{\partial s} \sum_{n_{1}, n_{2} \geq 0}\left(x+n_{1} \omega_{1}+n_{2} \omega_{2}\right)^{-s}\right|_{s=0}\right)
$$

It satisfies

$$
\frac{S_{2}\left(x+\omega_{1} \mid \omega_{1}, \omega_{2}\right)}{S_{2}\left(x \mid \omega_{1}, \omega_{2}\right)}=\frac{1}{2 \sin \frac{\pi x}{\omega_{2}}}, \quad \frac{S_{2}\left(x+\omega_{2} \mid \omega_{1}, \omega_{2}\right)}{S_{2}\left(x \mid \omega_{1}, \omega_{2}\right)}=\frac{1}{2 \sin \frac{\pi x}{\omega_{1}}} .
$$

$-S_{0}(\beta)$ is the limit of the normalisation factor $1 / \kappa\left(z^{2}\right)$ of the matrix (2.1). 
In the particular case $N=2$, one recovers explicitly [8]:

$$
R(\beta, r)=-S_{0}(\beta)\left(\begin{array}{cccc}
\frac{\cosh \frac{\beta}{2 r} \cosh \frac{i \pi}{2 r}}{\cosh \frac{i \pi-\beta}{2 r}} & 0 & 0 & -\frac{\sinh \frac{\beta}{2 r} \sinh \frac{i \pi}{2 r}}{\cosh \frac{i \pi-\beta}{2 r}} \\
0 & -\frac{\sinh \frac{\beta}{2 r} \cosh \frac{i \pi}{2 r}}{\sinh \frac{i \pi-\beta}{2 r}} & \frac{\cosh \frac{\beta}{2 r} \sinh \frac{i \pi}{2 r}}{\sinh \frac{i \pi-\beta}{2 r}} & 0 \\
0 & \frac{\cosh \frac{\beta}{2 r} \sinh \frac{i \pi}{2 r}}{\sinh \frac{i \pi-\beta}{2 r}} & -\frac{\sinh \frac{\beta}{2 r} \cosh \frac{i \pi}{2 r}}{\sinh \frac{i \pi-\beta}{2 r}} & 0 \\
-\frac{\sinh \frac{\beta}{2 r} \sinh \frac{i \pi}{2 r}}{\cosh \frac{i \pi-\beta}{2 r}} & 0 & 0 & \frac{\cosh \frac{\beta}{2 r} \cosh \frac{i \pi}{2 r}}{\cosh \frac{i \pi-\beta}{2 r}}
\end{array}\right) .
$$

Theorem 1 The matrix $R(\beta, r)$ satisfies the following properties:

- Yang-Baxter equation:

$$
R_{12}\left(\beta_{1}-\beta_{2}\right) R_{13}\left(\beta_{1}-\beta_{3}\right) R_{23}\left(\beta_{2}-\beta_{3}\right)=R_{23}\left(\beta_{2}-\beta_{3}\right) R_{13}\left(\beta_{1}-\beta_{3}\right) R_{12}\left(\beta_{1}-\beta_{2}\right),
$$

- Unitarity:

$$
R_{12}(\beta) R_{21}(-\beta)=1
$$

- Crossing-symmetry:

$$
R_{12}(\beta)^{t_{2}} R_{21}(-\beta+N i \pi)^{t_{2}}=1
$$

- Quasi-periodicity:

$$
\widehat{R}_{12}(\beta-i \pi r)=(h \otimes \mathbb{I})^{-1} \widehat{R}_{21}(-\beta)^{-1}(h \otimes \mathbb{I}),
$$

where

$$
\widehat{R}_{12}(\beta)=\frac{\sin \frac{\pi-i \beta}{N}}{\sin \frac{i \beta}{N}} R_{12}(\beta) .
$$

Note that the antisymmetry property of the elliptic $R$ matrix (2.1) under $z \rightarrow-z$ does not make sense in the scaling limit (2.12) for obvious reasons.

Proof: The proof of Theorem 1 is based on explicit computations using the form of the matrix (2.14) and standard arguments about meromorphic functions. Illustration is given on the example of the unitarity relation. We recall that the explicit form for the $R$ matrix is:

$$
R_{a, b}^{c, a+b-c}(\beta, r)=-\frac{1}{N} S_{0}(\beta) \frac{\sin \frac{i \beta}{r} \sin \frac{\pi}{r}}{\sin \frac{i \beta+\pi}{r}}\left(\operatorname{cotan} \frac{i \beta+(b-c) \pi r}{N r}-\operatorname{cotan} \frac{-\pi+(a-c) \pi r}{N r}\right)
$$

Denoting $x=-\pi / N r$ and $y=i \beta / N r$, eq. (2.21) reads:

$$
\begin{aligned}
R_{12}(x, y) R_{21}(x,-y)= & \mathcal{N} \sum_{a, b, c=1}^{N} \sum_{i=1}^{N}\left(\left(\operatorname{cotan}\left(y+\frac{(b-i) \pi}{N}\right)-\operatorname{cotan}\left(x+\frac{(a-i) \pi}{N}\right)\right)\right. \\
& \left.\left(-\operatorname{cotan}\left(x+\frac{(c-i) \pi}{N}\right)+\operatorname{cotan}\left(-y+\frac{(i-a-b+c) \pi}{N}\right)\right)\right) e_{a}^{c} \otimes e_{b}^{a+b-c},
\end{aligned}
$$


where $\mathcal{N}^{-1}=N^{2}\left(\operatorname{cotan}^{2} N x-\operatorname{cotan}^{2} N y\right)$; this overall factor comes from the product of the index-

independent factors $-\frac{1}{N} S_{0}(\beta) \frac{\sin \frac{i \beta}{r} \sin \frac{\pi}{r}}{\sin \frac{i \beta+\pi}{r}}$. Notice in particular that $S_{0}(\beta) S_{0}(-\beta)=1$ as obviously follows from (2.15).

We now prove that the coefficient function in (2.26) is proportional to $\delta_{a c}$ by analyzing its poles as a function of $y$.

If $a \neq c$, this function has simple poles at $y=k \pi / N$ for all integer $k$ since $a, b, c$ and $i$ are integers in (2.26). One immediately sees on its explicit expression that the residue is in fact zero. Hence, the function does not depend on $y$. One then evaluates it at $y=x+(a-b) \pi / N$ where it becomes zero.

If now $a=c$, this function can be directly computed. The relation (2.26) becomes:

$$
R_{12}(x, y) R_{21}(x,-y)=\mathcal{N} \sum_{a, b=1}^{N} \sum_{i=1}^{N}\left(\operatorname{cotan}^{2}\left(x+\frac{(a-i) \pi}{N}\right)-\operatorname{cotan}^{2}\left(y+\frac{(b-i) \pi}{N}\right)\right) e_{a}^{a} \otimes e_{b}^{b} .
$$

Since the sum over $i$ is manifestly cyclic, the expression does not depend on $a$ and $b$. It is then explicitly evaluated from the equality [25]

$$
\sum_{i} \operatorname{cotan}^{2}(x+i \pi / N)=N^{2}-N+N^{2} \operatorname{cotan}^{2} N x
$$

Combining (2.27), (2.28) and the expression of the normalisation factor $\mathcal{N}$, one gets the desired result.

The crossing-symmetry (2.22) is proved by similar arguments. The quasi-periodicity relation (2.23) is essentially trivial to prove. The Yang-Baxter equation by contrast requires a careful analysis of the behaviour at the poles, involving as it does combinations of double and simple poles.

Remark: Using the crossing symmetry and the unitarity properties of $R_{12}$, one can exchange inversion and transposition for the matrix $R_{12}$ as follows (the same property also holds for the matrix $\left.\widehat{R}_{12}\right)$ :

$$
\left(R_{12}(\beta)^{t_{2}}\right)^{-1}=\left(R_{12}(\beta-N i \pi)^{-1}\right)^{t_{2}}
$$

\subsection{Definition of the algebra}

The limit of the elliptic quantum algebra structure $\mathcal{A}_{q, p}\left(\widehat{s l}(N)_{c}\right)$ defined by the scaling procedure (2.12) leads to an algebraic structure parametrised by the limit $R$-matrix for abstract generators $L(\beta)$ encapsulated into a $N \times N$ matrix

$$
L(\beta)=\left(\begin{array}{ccc}
L_{11}(\beta) & \cdots & L_{1 N}(\beta) \\
\vdots & & \vdots \\
L_{N 1}(\beta) & \cdots & L_{N N}(\beta)
\end{array}\right) .
$$

One defines $\mathcal{D} Y_{r}(g l(N))_{c}$ by imposing the following constraints on the $L_{i j}(\beta)$ (with the matrix $\widehat{R}_{12}$ given by eq. (2.24)):

$$
\widehat{R}_{12}\left(\beta_{1}-\beta_{2}\right) L_{1}\left(\beta_{1}\right) L_{2}\left(\beta_{2}\right)=L_{2}\left(\beta_{2}\right) L_{1}\left(\beta_{1}\right) \widehat{R}_{12}^{*}\left(\beta_{1}-\beta_{2}\right),
$$


where $L_{1}(\beta) \equiv L(\beta) \otimes \mathbb{I}, L_{2}(\beta) \equiv \mathbb{I} \otimes L(\beta)$ and $\widehat{R}_{12}^{*}$ is defined by $\widehat{R}_{12}^{*}(\beta, r) \equiv \widehat{R}_{12}(\beta, r-c)$.

The matrix $\widehat{R}_{12}^{*}$ obeys also the unitarity, crossing-symmetry and quasi-periodicity conditions of Theorem 1.

The $q$-determinant $q$-det $L(\beta)$ is given by $(\varepsilon(\sigma)$ being the signature of the permutation $\sigma)$

$$
q-\operatorname{det} L(\beta) \equiv \sum_{\sigma \in \mathfrak{S}_{N}} \varepsilon(\sigma) \prod_{k=1}^{N} L_{k, \sigma(k)}(\beta-i \pi(k-N-1)) .
$$

It lies in the centre of $\mathcal{D} Y_{r}(g l(N))_{c}$; it can be "factored out" and set to the value 1 so as to get

$$
\mathcal{D} Y_{r}(\operatorname{sl}(N))_{c}=\mathcal{D} Y_{r}(g l(N))_{c} /\langle q-\operatorname{det} L-1\rangle .
$$

By analogy with the full elliptic case we now introduce the following two matrices:

$$
\begin{aligned}
& L^{+}(\beta) \equiv L\left(\beta-\frac{1}{2} i \pi c\right), \\
& L^{-}(\beta) \equiv h L(\beta-i \pi r) h^{-1} .
\end{aligned}
$$

They obey coupled exchange relations following from (2.31) and periodicity/unitarity properties of the matrices $\widehat{R}_{12}$ and $\widehat{R}_{12}^{*}$ :

$$
\begin{aligned}
& \widehat{R}_{12}\left(\beta_{1}-\beta_{2}\right) L_{1}^{ \pm}\left(\beta_{1}\right) L_{2}^{ \pm}\left(\beta_{2}\right)=L_{2}^{ \pm}\left(\beta_{2}\right) L_{1}^{ \pm}\left(\beta_{1}\right) \widehat{R}_{12}^{*}\left(\beta_{1}-\beta_{2}\right), \\
& \widehat{R}_{12}\left(\beta_{1}-\beta_{2}-\frac{1}{2} i \pi c\right) L_{1}^{+}\left(\beta_{1}\right) L_{2}^{-}\left(\beta_{2}\right)=L_{2}^{-}\left(\beta_{2}\right) L_{1}^{+}\left(\beta_{1}\right) \widehat{R}_{12}^{*}\left(\beta_{1}-\beta_{2}+\frac{1}{2} i \pi c\right) .
\end{aligned}
$$

Important remark: The algebraic structure (2.35) and the properties $(2.20)-(2.23)$ and $(2.29)$ are identical to the algebraic structure and properties of $R$-matrices for the full elliptic case, provided that the parameters $p, z$ be replaced by their scaling limits $r, \beta$; the multiplicative shifts are replaced by additive shifts; and the scalar factor in $\widehat{R}$ be replaced by its renormalised scaling limit (2.24). It follows that all conclusions touching the existence and structure functions of algebraic substructures (exchange algebras; Abelian algebras and Poisson algebras) can be transposed from the elliptic case to the scaling limit by suitable changes of the structure functions, provided that their proofs in 20, 21 use only the relations equivalent to (2.20 2.23), (2.29) and (2.35).

\section{Exchange algebras on the double Yangian $\mathcal{D} Y_{r}(s l(N))_{c}$}

Lemma 1 Let $\mathcal{L}^{(M)}(\beta)=\left(h^{-M} L^{+}\left(\beta-\frac{1}{2} i \pi c\right)\right)^{t} \widetilde{L}^{-}(\beta)$, where $\widetilde{L}^{ \pm}(\beta) \equiv\left(L^{ \pm}(\beta)^{t}\right)^{-1}$ and $M \in \mathbb{Z}$. The operators $\mathcal{L}(\beta)$ have the following exchange properties with the generators $L$ of $\mathcal{D} Y_{r}(\operatorname{sl}(N))_{c}$ when the parameters $c$ and $r$ obey the relation $c=-N-M r$ :

$$
\mathcal{L}_{1}^{(M)}\left(\beta_{1}\right) L_{2}\left(\beta_{2}\right)=F\left(M, \beta_{2}-\beta_{1}\right) L_{2}\left(\beta_{2}\right)\left(\widehat{R}_{21}^{*}\left(\beta_{2}-\beta_{1}+i \pi c\right)^{-1}\right)^{t_{1}} \mathcal{L}_{1}^{(M)}\left(\beta_{1}\right) \widehat{R}_{21}^{*}\left(\beta_{2}-\beta_{1}+i \pi c\right)^{t_{1}}
$$

where

$$
F(M, \beta)= \begin{cases}\prod_{k=0}^{M-1} \frac{\sin ^{2} \frac{i \beta+k \pi r}{N}}{\sin \frac{i \beta+k \pi r+\pi}{N} \sin \frac{i \beta+k \pi r-\pi}{N}} & \text { for } M>0, \\ \prod_{k=1}^{|M|} \frac{\sin \frac{-i \beta+k \pi r+\pi}{N} \sin \frac{-i \beta+k \pi r-\pi}{N}}{\sin ^{2} \frac{-i \beta+k \pi r}{N}} & \text { for } M<0,\end{cases}
$$


and

$$
F(0, \beta)=1 .
$$

We give here an extensive proof of Lemma 1 as an illustration of the transposition procedure given in the previous remark. Other proof will simply call back to this key feature.

Proof: Let us prove the lemma in terms of $L^{+}(\beta)$. One has

$$
\mathcal{L}_{1}^{(M)}\left(\beta_{1}\right) L_{2}^{+}\left(\beta_{2}\right)=L_{1}^{+}\left(\beta_{1}-\frac{1}{2} i \pi c\right)^{t_{1}}\left(h_{1}^{-M}\right)^{t_{1}} \widetilde{L}_{1}^{-}\left(\beta_{1}\right) L_{2}^{+}\left(\beta_{2}\right) .
$$

Exchanging $\mathcal{L}_{1}^{(M)}\left(\beta_{1}\right)$ with $L_{2}^{+}\left(\beta_{2}\right)$ requires the following relations obtained from (2.35):

$$
\begin{aligned}
\left(\widehat{R}_{21}\left(\beta_{2}-\beta_{1}-\frac{1}{2} i \pi c\right)^{t_{1}}\right)^{-1} L_{2}^{+}\left(\beta_{2}\right) \widetilde{L}_{1}^{-}\left(\beta_{1}\right) & =\widetilde{L}_{1}^{-}\left(\beta_{1}\right) L_{2}^{+}\left(\beta_{2}\right)\left(\widehat{R}_{21}^{*}\left(\beta_{2}-\beta_{1}+\frac{1}{2} i \pi c\right)^{t_{1}}\right)^{-1} \\
L_{1}^{+}\left(\beta_{1}\right)^{t_{1}}\left(\widehat{R}_{21}\left(\beta_{2}-\beta_{1}\right)^{-1}\right)^{t_{1}} L_{2}^{+}\left(\beta_{2}\right) & =L_{2}^{+}\left(\beta_{2}\right)\left(\widehat{R}_{21}^{*}\left(\beta_{2}-\beta_{1}\right)^{-1}\right)^{t_{1}} L_{1}^{+}\left(\beta_{1}\right)^{t_{1}}
\end{aligned}
$$

Using (3.5a), one gets

$$
\begin{aligned}
\mathcal{L}_{1}^{(M)}\left(\beta_{1}\right) L_{2}^{+}\left(\beta_{2}\right)= & L_{1}^{+}\left(\beta_{1}-\frac{1}{2} i \pi c\right)^{t_{1}}\left(h_{1}^{-M}\right)^{t_{1}}\left(\widehat{R}_{21}\left(\beta_{2}-\beta_{1}-\frac{1}{2} i \pi c\right)^{t_{1}}\right)^{-1} \\
& L_{2}^{+}\left(\beta_{2}\right) \widetilde{L}_{1}^{-}\left(\beta_{1}\right) \widehat{R}_{21}^{*}\left(\beta_{2}-\beta_{1}+\frac{1}{2} i \pi c\right)^{t_{1}} \\
= & L_{1}^{+}\left(\beta_{1}-\frac{1}{2} i \pi c\right)^{t_{1}}\left(h_{1}^{-M}\right)^{t_{1}}\left(\widehat{R}_{21}\left(\beta_{2}-\beta_{1}-\frac{1}{2} i \pi c\right)^{t_{1}}\right)^{-1} \\
& \left(h_{1}^{M}\right)^{t_{1}} L_{2}^{+}\left(\beta_{2}\right)\left(h_{1}^{-M}\right)^{t_{1}} \widetilde{L}_{1}^{-}\left(\beta_{1}\right) \widehat{R}_{21}^{*}\left(\beta_{2}-\beta_{1}+\frac{1}{2} i \pi c\right)^{t_{1}} .
\end{aligned}
$$

From the crossing unitarity property (2.29) of $\widehat{R}_{21}$, this equation can be written as

$$
\begin{gathered}
\mathcal{L}_{1}^{(M)}\left(\beta_{1}\right) L_{2}^{+}\left(\beta_{2}\right)=L_{1}^{+}\left(\beta_{1}-\frac{1}{2} i \pi c\right)^{t_{1}}\left(h_{1}^{M} \widehat{R}_{21}\left(\beta_{2}-\beta_{1}-\frac{1}{2} i \pi c-N i \pi\right)^{-1} h_{1}^{-M}\right)^{t_{1}} \\
L_{2}^{+}\left(\beta_{2}\right)\left(h_{1}^{-M}\right)^{t_{1}} \widetilde{L}_{1}^{-}\left(\beta_{1}\right) \widehat{R}_{21}^{*}\left(\beta_{2}-\beta_{1}+\frac{1}{2} i \pi c\right)^{t_{1}} .
\end{gathered}
$$

Now sufficient conditions that allow substitution of eq. (3.5b) into (3.7) are

$$
M r=-c-N \quad \text { where } \quad M \in \mathbb{Z} .
$$

At this point, it is necessary to distinguish whether $M=0$ or not.

- In the case $M=0$, the condition (3.8) fixes the central charge $c$ to the critical value $-N$. The argument of $\widehat{R}_{21}$ in eq. (3.7) is then exactly the one needed to use eq. (3.5b). Hence inserting (3.5b) into (3.7), one obtains (with $\mathcal{L}(\beta) \equiv \mathcal{L}^{(0)}(\beta)$ )

$$
\begin{aligned}
& \mathcal{L}_{1}\left(\beta_{1}\right) L_{2}^{+}\left(\beta_{2}\right)= \\
& \quad=L_{2}^{+}\left(\beta_{2}\right)\left(\widehat{R}_{21}^{*}\left(\beta_{2}-\beta_{1}+\frac{1}{2} i \pi c\right)^{-1}\right)^{t_{1}} L_{1}^{+}\left(\beta_{1}-\frac{1}{2} i \pi c\right)^{t_{1}} \widetilde{L}_{1}^{-}\left(\beta_{1}\right) \widehat{R}_{21}^{*}\left(\beta_{2}-\beta_{1}+\frac{1}{2} i \pi c\right)^{t_{1}} \\
& \quad=L_{2}^{+}\left(\beta_{2}\right)\left(\widehat{R}_{21}^{*}\left(\beta_{2}-\beta_{1}+\frac{1}{2} i \pi c\right)^{-1}\right)^{t_{1}} \mathcal{L}_{1}\left(\beta_{1}\right) \widehat{R}_{21}^{*}\left(\beta_{2}-\beta_{1}+\frac{1}{2} i \pi c\right)^{t_{1}}
\end{aligned}
$$

which is formula (3.1).

This proof reproduces exactly the proof given in [20, 21] for the identification of exchange properties in the elliptic case. The relation $c=-N-M r$ is the scaling limit of the relation $p^{M / 2}=q^{-c-N}$ in [21]. Identification of the relations (2.20)-(2.23) with scaling limits of [21] is crucial in this procedure. 
- In the case $M \neq 0$, the argument of $\widehat{R}_{21}$ in eq. (3.7) is $\beta_{2}-\beta_{1}+\frac{1}{2} i \pi c+i \pi M r$. The use of the condition (3.8) thus relies on the quasiperiodicity property of $\widehat{R}_{21}$. More precisely, one has

$$
h_{1}^{M} \widehat{R}_{21}(\beta+i \pi M r) h_{1}^{-M}=F(-M, \beta) \widehat{R}_{21}(\beta) .
$$

Then on the line defined by (3.8), the equation (3.7) becomes

$$
\begin{aligned}
\mathcal{L}_{1}^{(M)}\left(\beta_{1}\right) L_{2}^{+}\left(\beta_{2}\right)= & F\left(-M, \beta_{2}-\beta_{1}+\frac{1}{2} i \pi c\right)^{-1} L_{1}^{+}\left(\beta_{1}-\frac{1}{2} i \pi c\right)^{t_{1}}\left(\widehat{R}_{21}\left(\beta_{2}-\beta_{1}+\frac{1}{2} i \pi c\right)^{-1}\right)^{t_{1}} \\
& L_{2}^{+}\left(\beta_{2}\right)\left(h_{1}^{-M}\right)^{t_{1}} \widetilde{L}_{1}^{-}\left(\beta_{1}\right) \widehat{R}_{21}^{*}\left(\beta_{2}-\beta_{1}+\frac{1}{2} i \pi c\right)^{t_{1}} \\
= & F\left(-M, \beta_{2}-\beta_{1}+\frac{1}{2} i \pi c\right)^{-1} L_{2}^{+}\left(\beta_{2}\right)\left(\widehat{R}_{21}^{*}\left(\beta_{2}-\beta_{1}+\frac{1}{2} i \pi c\right)^{-1}\right)^{t_{1}} \\
& L_{1}^{+}\left(\beta_{1}-\frac{1}{2} i \pi c\right)^{t_{1}}\left(h_{1}^{-M}\right)^{t_{1}} \widetilde{L}_{1}^{-}\left(\beta_{1}\right) \widehat{R}_{21}^{*}\left(\beta_{2}-\beta_{1}+\frac{1}{2} i \pi c\right)^{t_{1}}
\end{aligned}
$$

the last equality following from eq. (3.5b). Hence we get, with $\beta=\beta_{2}-\beta_{1}+\frac{1}{2} i \pi c$,

$$
\mathcal{L}_{1}^{(M)}\left(\beta_{1}\right) L_{2}^{+}\left(\beta_{2}\right)=F(-M, \beta)^{-1} L_{2}^{+}\left(\beta_{2}\right)\left(\widehat{R}_{21}^{*}(\beta)^{-1}\right)^{t_{1}} \mathcal{L}_{1}^{(M)}\left(\beta_{1}\right) \widehat{R}_{21}^{*}(\beta)^{t_{1}} .
$$

Finally, one can check that $F(-M, \beta)^{-1}=F(M, \beta-i \pi c)$, which achieves the proof.

In the case $N=2$, the formula (3.2) reduces to

$$
F(M, \beta)= \begin{cases}(-1)^{M} \prod_{k=0}^{M-1} \tan ^{2} \frac{i \beta+k \pi r}{2} & \text { for } M>0, \\ (-1)^{M} \prod_{k=1}^{|M|} \operatorname{cotan}^{2} \frac{i \beta-k \pi r}{2} & \text { for } M<0 .\end{cases}
$$

\subsection{Centre of $\mathcal{D} Y_{r}(s l(N))_{c}$ at the critical level $c=-N$}

Using the previous lemma, it is straightforward to prove the following theorem:

Theorem 2 At the critical level $c=-N$, the operators generated by

$$
t(\beta) \equiv \operatorname{Tr}[\mathcal{L}(\beta)] \equiv \operatorname{Tr}\left(L^{+}\left(\beta-\frac{1}{2} i \pi c\right)^{t} \widetilde{L}^{-}(\beta)\right)
$$

lie in the centre of the algebra $\mathcal{D} Y_{r}(s l(N))_{c}$.

Proof: Equation (3.1) leads to

$$
\begin{aligned}
t\left(\beta_{1}\right) L_{2}\left(\beta_{2}\right) & =\operatorname{Tr}_{1}\left[\mathcal{L}_{1}\left(\beta_{1}\right)\right] L_{2}\left(\beta_{2}\right) \\
& =L_{2}\left(\beta_{2}\right) \operatorname{Tr}_{1}\left[\left(\widehat{R}_{21}^{*}\left(\beta_{2}-\beta_{1}+i \pi c\right)^{-1}\right)^{t_{1}} \mathcal{L}_{1}\left(\beta_{1}\right) \widehat{R}_{21}^{*}\left(\beta_{2}-\beta_{1}+i \pi c\right)^{t_{1}}\right] .
\end{aligned}
$$

Using the fact that under a trace over the space 1 one has $\operatorname{Tr}_{1}\left(R_{21} \mathcal{L}_{1} R^{\prime}{ }_{21}\right)=\operatorname{Tr}_{1}\left(\mathcal{L}_{1} R_{21}^{\prime t_{2}} R_{21}{ }^{t_{2}}\right)^{t_{2}}$, one gets

$$
t\left(\beta_{1}\right) L_{2}\left(\beta_{2}\right)=L_{2}\left(\beta_{2}\right) \operatorname{Tr}_{1}\left[\mathcal{L}_{1}\left(\beta_{1}\right) \widehat{R}_{21}^{*}\left(\beta_{2}-\beta_{1}+i \pi c\right)^{t_{1} t_{2}}\left(\widehat{R}_{21}^{*}\left(\beta_{2}-\beta_{1}+i \pi c\right)^{-1}\right)^{t_{1} t_{2}}\right]^{t_{2}} .
$$


The last two terms in the right hand side cancel each other, leaving a trivial dependence in space 2 and $\operatorname{Tr}_{1} \mathcal{L}_{1}\left(\beta_{1}\right) \equiv t\left(\beta_{1}\right)$ in space 1 . This shows the commutation of $t\left(\beta_{1}\right)$ with $L\left(\beta_{2}\right)$, that is with the full algebra $\mathcal{D} Y_{r}(\operatorname{sl}(N))_{c}$ at $c=-N$.

Corollary 1 At the critical level $c=-N$ the operators $t(\beta)$ realise an Abelian algebra

$$
t\left(\beta_{1}\right) t\left(\beta_{2}\right)=t\left(\beta_{2}\right) t\left(\beta_{1}\right) \text {. }
$$

The proof is obvious from the previous theorem.

\subsection{Quantum exchange algebras}

Lemma 2 When the parameters $r$ and $c$ satisfy the relation $M r=-c-N$, with $M \in \mathbb{Z}$, the operators $t(\beta)=\operatorname{Tr}\left[\mathcal{L}^{(M)}(\beta)\right]$ realise an exchange algebra with the generators $L$ of $\mathcal{D} Y_{r}(\operatorname{sl}(N))_{c}$ :

$$
t\left(\beta_{1}\right) L\left(\beta_{2}\right)=F\left(M, \beta_{2}-\beta_{1}\right) L\left(\beta_{2}\right) t\left(\beta_{1}\right)
$$

the function $F(M, \beta)$ being given by (3.9).

Proof: The proof follows the lines of that of Theorem 2, starting from relation (3.1) with $M \neq 0$.

Theorem 3 Under the condition $M r=-c-N$, with $M \in \mathbb{Z}$, the operators $t(\beta)=\operatorname{Tr}\left[\mathcal{L}^{(M)}(\beta)\right]$ close a quadratic algebra

$$
t\left(\beta_{1}\right) t\left(\beta_{2}\right)=\mathcal{Y}_{N, r, M}\left(\beta_{2}-\beta_{1}\right) t\left(\beta_{2}\right) t\left(\beta_{1}\right)
$$

where the function $\mathcal{Y}$ is given by

$$
\mathcal{Y}_{N, r, M}(\beta)=\left\{\begin{array}{lll}
\prod_{k=1}^{M} \frac{\sin ^{2} \frac{i \beta-k \pi r}{N}}{\sin ^{2} \frac{i \beta+k \pi r}{N}} \frac{\sin \frac{i \beta+k \pi r+\pi}{N}}{\sin \frac{i \beta-k \pi r+\pi}{N}} \frac{\sin \frac{i \beta+k \pi r-\pi}{N}}{\sin \frac{i \beta-k \pi r-\pi}{N}} & \text { for } M>0, \\
\prod_{k=1}^{|M|-1} \frac{\sin ^{2} \frac{i \beta-k \pi r}{N}}{\sin ^{2} \frac{i \beta+k \pi r}{N}} & \frac{\sin \frac{i \beta+k \pi r+\pi}{N}}{\sin \frac{i \beta-k \pi r+\pi}{N}} \frac{\sin \frac{i \beta+k \pi r-\pi}{N}}{\sin \frac{i \beta-k \pi r-\pi}{N}} & \text { for } M<0 .
\end{array}\right.
$$

Proof: Lemma 2 and definitions (2.34) imply that

$$
\begin{aligned}
t\left(\beta_{1}\right) L^{+}\left(\beta_{2}\right) & =F\left(M, \beta_{2}-\beta_{1}-\frac{1}{2} i \pi c\right) L^{+}\left(\beta_{2}\right) t\left(\beta_{1}\right) \\
t\left(\beta_{1}\right) L^{-}\left(\beta_{2}\right)^{-1} & =F\left(M, \beta_{2}-\beta_{1}-i \pi r\right)^{-1} L^{-}\left(\beta_{2}\right)^{-1} t\left(\beta_{1}\right) .
\end{aligned}
$$

Since $t(\beta)=\operatorname{Tr}\left[\mathcal{L}^{(M)}(\beta)\right]=\operatorname{Tr}\left[\left(h^{-M} L^{+}\left(\beta-\frac{1}{2} i \pi c\right)\right)^{t}\left(L^{-}(\beta)^{-1}\right)^{t}\right]$, one gets

$$
t\left(\beta_{1}\right) t\left(\beta_{2}\right)=\frac{F\left(M, \beta_{2}-\beta_{1}-i \pi c\right)}{F\left(M, \beta_{2}-\beta_{1}-i \pi r\right)} t\left(\beta_{2}\right) t\left(\beta_{1}\right) .
$$

The function $\mathcal{Y}_{N, r, M}\left(\beta_{2}-\beta_{1}\right)$ being defined by the above ratio of $F$ functions, its explicit expression (3.20) is obtained from formula (3.2). 
When $N=2$, the expression $(3.20)$ becomes

$$
\mathcal{Y}_{2, r, M}(\beta)= \begin{cases}\prod_{k=1}^{M} \frac{\tan ^{2} \frac{i \beta-k \pi r}{2}}{\tan ^{2} \frac{i \beta+k \pi r}{2}} & \text { for } M>0, \\ \prod_{k=1}^{|M|-1} \frac{\tan ^{2} \frac{i \beta-k \pi r}{2}}{\tan ^{2} \frac{i \beta+k \pi r}{2}} & \text { for } M<0 .\end{cases}
$$

Corollary 2 When both relations $M r=-c-N$ and $2 r=N h$ hold, with $M, h \in \mathbb{Z}$, the function $\mathcal{Y}_{N, r, M}(\beta)$ is equal to one. The operators $t(\beta)$ therefore generate an Abelian algebra.

Proof: Direct calculation.

\section{Poisson structures on $\mathcal{D} Y_{r}(s l(N))_{c}$}

The results of the previous section have shown that the operators $t(\beta)$ generate an Abelian subalgebra of $\mathcal{D} Y_{r}(s l(N))_{c}$ when certain conditions on the parameters $c, r$ are fulfilled. One can then naturally induce a Poisson structure on this Abelian algebra by considering the exchange relations between the $t(\beta)$ in the neighbourhood of the critical line $c=-N$ or the lines $M r=-c-N$ eq. (3.8).

\subsection{Poisson structure on the centre at $c=-N$}

Theorem 4 The Poisson structure of the generators $t(\beta)$ around $c=-N$ reads:

$$
\left\{t\left(\beta_{1}\right), t\left(\beta_{2}\right)\right\}=\frac{-\frac{2 \pi}{N} \sin ^{2} \frac{\pi}{N} \cos \frac{i\left(\beta_{2}-\beta_{1}\right)}{N}}{\sin \frac{i\left(\beta_{2}-\beta_{1}\right)}{N} \sin \frac{i\left(\beta_{2}-\beta_{1}\right)+\pi}{N} \sin \frac{i\left(\beta_{2}-\beta_{1}\right)-\pi}{N}} t\left(\beta_{1}\right) t\left(\beta_{2}\right) .
$$

\section{Proof:}

The proof follows the same scheme as the computation of the Poisson structures in the elliptic case at critical value $c=-N$. We briefly recall the basic steps:

- Step 1: The exchange algebra for the generators $t(\beta)$ around $c=-N$ reads

$$
t\left(\beta_{1}\right) t\left(\beta_{2}\right)=T\left(\beta_{2}-\beta_{1}\right) \mathcal{M}\left(\beta_{2}-\beta_{1}\right)_{j_{1} j_{2}}^{i_{1} i_{2}} \mathcal{L}\left(\beta_{2}\right)_{i_{2}}^{j_{2}} \quad \mathcal{L}\left(\beta_{1}\right)_{i_{1}}^{j_{1}}
$$

where

$$
\mathcal{M}(\beta)=\left(\left(R_{21}(\beta) R_{21}(\beta-i \pi c-i \pi N)^{-1} R_{12}(-\beta)^{-1}\right)^{t_{2}} R_{12}(-\beta-i \pi c)^{t_{2}}\right)^{t_{2}}
$$

and

$$
T(\beta)=\frac{\sin \frac{i \beta-\pi}{N}}{\sin \frac{i \beta+\pi}{N}} \frac{\sin \frac{i \beta+\pi-\pi c}{N}}{\sin \frac{i \beta-\pi+\pi c}{N}} \frac{\sin \frac{i \beta+\pi c}{N}}{\sin \frac{i \beta-\pi c}{N}} .
$$


- Step 2: At $c=-N$, the derivative of $\mathcal{M}(\beta)$ with respect to $c$ vanishes due to the crossing-unitarity relation (it can actually be identified at $c=-N$ with the derivative of the crossing-unitarity relation (2.29) with respect to the spectral parameter). Hence, the derivative of the exchange relation at $c=-N$ closes a Poisson structure on $t\left(\beta_{1}\right)$.

- Step 3: The Poisson structure function is now given by the derivative with respect to $c$ of the factor $T$ in eq. (4.4). This factor is a product of the scalar normalisers used to rescale the structure $R$-matrix $\widehat{R}$ with respect to the unitary $R$-matrix $R$ in (2.24). Equation (4.1) immediately follows.

\subsection{Poisson structure on the lines}

Theorem 5 Setting $N h=2 r+\epsilon$, for any non zero integer $h$, one defines the $h$-labelled Poisson structure by

$$
\left\{t\left(\beta_{1}\right), t\left(\beta_{2}\right)\right\}_{h}=\lim _{\epsilon \rightarrow 0} \frac{1}{\epsilon}\left(t\left(\beta_{1}\right) t\left(\beta_{2}\right)-t\left(\beta_{2}\right) t\left(\beta_{1}\right)\right) .
$$

Its explicit expression is

$$
\left\{t\left(\beta_{1}\right), t\left(\beta_{2}\right)\right\}_{h}=f_{h}\left(\beta_{2}-\beta_{1}\right) t\left(\beta_{1}\right) t\left(\beta_{2}\right)
$$

where

$$
f_{h}(\beta)= \begin{cases}M(M+1) f_{s}(\beta) & \text { for } h \text { even } \\ 2 E\left(\frac{M}{2}\right)\left(E\left(\frac{M}{2}\right)+1\right) f_{s}(\beta)+2 E\left(\frac{M+1}{2}\right)^{2} f_{c}(\beta) & \text { for } h \text { odd }\end{cases}
$$

with

and

$$
f_{s}(\beta)=\frac{-\frac{\pi}{N} \sin ^{2} \frac{\pi}{N} \cos \frac{i \beta}{N}}{\sin \frac{i \beta}{N} \sin \frac{i \beta+\pi}{N} \sin \frac{i \beta-\pi}{N}}
$$

$$
f_{c}(\beta)=\frac{\frac{\pi}{N} \sin ^{2} \frac{\pi}{N} \sin \frac{i \beta}{N}}{\cos \frac{i \beta}{N} \cos \frac{i \beta+\pi}{N} \cos \frac{i \beta-\pi}{N}}
$$

Proof: By direct calculation, after noting that the right hand side of (4.5) is equal to $\left.\frac{d \mathcal{Y}}{d \epsilon}\right|_{\epsilon=0} t\left(\beta_{1}\right) t\left(\beta_{2}\right)$.

In the case $N=2$, the functions $f_{s}$ and $f_{c}$ take the simple form

$$
f_{s}(\beta)=-f_{c}(\beta)=\frac{\pi}{\sin i \beta} .
$$

Therefore there is only one type of Poisson structure in the $\operatorname{sl}(2)$ case since the normalisation factors in the r.h.s. of (4.7) may always be reabsorbed in the definition of $\epsilon$.

\section{$5 \quad$ Higher spin generators}

Theorem 6 We define the operators $w_{s}(\beta)(s=1, \ldots, N-1)$ by:

$$
w_{s}(\beta) \equiv \operatorname{Tr}\left[\prod_{1 \leq k \leq s}^{\curvearrowleft}\left(\prod_{j>k} P_{k j}\right) \prod_{1 \leq k \leq s}^{\curvearrowleft}\left(\mathcal{L}_{k}^{(M)}\left(\beta_{k}\right) \prod_{j>k} \widehat{R}_{k j}^{*}\left(\beta_{k}-\beta_{j}+i \pi N\right)^{t_{k} t_{j}}\right)\right]
$$


where

$$
\mathcal{L}_{k}^{(M)}(\beta) \equiv\left(h^{-M} L_{k}^{+}\left(\beta-\frac{1}{2} i \pi c\right)\right)^{t_{k}} \widetilde{L}_{k}^{-}(\beta) \equiv \underbrace{\mathbb{I} \otimes \ldots \otimes \mathbb{I}}_{k-1} \otimes\left(h^{-M} L^{+}\left(\beta-\frac{1}{2} i \pi c\right)\right)^{t} \widetilde{L}^{-}(\beta) \otimes \underbrace{\mathbb{I} \otimes \ldots \mathbb{I}}_{s-k}
$$

with $M \in \mathbb{Z}, \beta_{k}=\beta-i \pi\left(k-\frac{s+1}{2}\right)$, and $P_{k j}$ is the permutation operator between the spaces $k$ and $j$ including the spectral parameters.

On the lines $c=-N-M r$, the operators $w_{s}(\beta)$ realise an exchange algebra with the generators $L\left(\beta^{\prime}\right)$ of $\mathcal{D} Y_{r}(s l(N))_{c}$ :

$$
w_{s}(\beta) L\left(\beta^{\prime}\right)=F^{(s)}\left(M, \beta^{\prime}-\beta\right) L\left(\beta^{\prime}\right) w_{s}(\beta),
$$

where

$$
F^{(s)}\left(M, \beta^{\prime}-\beta\right)=\prod_{k=1}^{s} F\left(M, \beta^{\prime}-\beta_{k}\right) .
$$

Proof: The proof of Theorem 6 is based on the same algebraic arguments as in the case of the full elliptic algebra (see Theorem 6 of ref. [21]). Namely, the exchange relation (5.13) thus follows from the basic relation (3.1), the Yang-Baxter equation and the crossing-symmetry property (2.22).

One obtains the following corollary, the proof being obvious:

Corollary 3 On the lines $c=-N-M r$, the operators $w_{s}(\beta)$ realise an exchange algebra

$$
w_{s}(\beta) w_{s^{\prime}}\left(\beta^{\prime}\right)=\prod_{u=-\frac{s-1}{2}}^{\frac{s-1}{2}} \prod_{v=-\frac{s^{\prime}-1}{2}}^{\frac{s^{\prime}-1}{2}} \mathcal{Y}_{N, r, M}\left(\beta^{\prime}-\beta+i \pi(u-v)\right) w_{s^{\prime}}\left(\beta^{\prime}\right) w_{s}(\beta) .
$$

When a additional relation $2 r=N h$ with $h \in \mathbb{Z} \backslash\{0\}$ is imposed, the function $\mathcal{Y}_{N, r, M}$ is equal to 1 : the operators $w_{s}(\beta)$ realise then an Abelian subalgebra in $\mathcal{D} Y_{r}(s l(N))_{c}$.

The previous corollary allows us to define Poisson structures on the corresponding Abelian subalgebras. As usual, they are obtained as limits of the exchange algebras when $N h=2 r$ with $h \in \mathbb{Z} \backslash\{0\}$ :

Theorem 7 Setting $N h=2 r+\epsilon$ for any integer $h \neq 0$, the $h$-labelled Poisson structure defined by:

$$
\left\{w_{s}(\beta), w_{s^{\prime}}\left(\beta^{\prime}\right)\right\}_{(h)}=\lim _{\epsilon \rightarrow 0} \frac{1}{\epsilon}\left(w_{s}(\beta) w_{s^{\prime}}\left(\beta^{\prime}\right)-w_{s^{\prime}}\left(\beta^{\prime}\right) w_{s}(\beta)\right)
$$

has the following expression:

$$
\left\{w_{s}(\beta), w_{s^{\prime}}\left(\beta^{\prime}\right)\right\}=\sum_{u=-(s-1) / 2}^{(s-1) / 2} \sum_{v=-\left(s^{\prime}-1\right) / 2}^{\left(s^{\prime}-1\right) / 2} f_{h}\left(\beta^{\prime}-\beta+i \pi(u-v)\right) w_{s}(\beta) w_{s^{\prime}}\left(\beta^{\prime}\right),
$$

where $f_{h}(\beta)$ is given by (4.7).

Proof: The proof is made by direct calculation. 


\section{Conclusion}

We have proved that the existence of exchange subalgebras, Abelian subalgebras and the Poisson structures on these objects, all survived in the scaling limit $q \rightarrow 1, \frac{\ln p}{\ln q} \rightarrow r, \frac{\ln z}{\ln q} \rightarrow \frac{i \beta}{\pi}$, and the characteristic relations and structure functions were given by the scaling limits, suitably defined whenever potential divergences arise, of their analogues in the full elliptic case.

Two directions should now be investigated. We have already commented upon the necessary careful study of precise mode expansions of the generating functionals and structure function, required to construct explicit representations of such algebras.

In a more abstract setting, the question arises of interpreting more precisely the "deformation" involved when going from $\mathcal{D} Y(\operatorname{sl}(N))_{c}($ at $r=\infty)$ to $\mathcal{D} Y_{r}(\operatorname{sl}(N))_{c}$. We have established at $N=$ 2 that this deformation is in fact a Drinfel'd twist generated by the $q \rightarrow 1$ scaling limit of the twist $\mathcal{U}_{q}\left(\widehat{s l}(2)_{c}\right) \rightarrow \mathcal{A}_{q, p}\left(\widehat{s l}(2)_{c}\right)$ [5]. We shall report on this and other very interesting connections established between various algebraic objects of similar type [26.

\section{Acknowledgements}

This work was supported in part by CNRS and EC network contract number FMRX-CT96-0012. M.R. was supported by an EPSRC research grant no. GR/K 79437. The work greatly benefitted from penetrating observations and suggestions of P. Sorba. The authors would like to thank V. Korepin for his stimulating questions and S.M. Khoroshkin and H. Konno for valuable clarifications. J.A. and M.R. wish to thank the LAPTH for its kind hospitality.

\section{References}

[1] E. K. Sklyanin, Some algebraic structures connected with the Yang-Baxter equation, Funct. Anal. Appl. 16 (1982) 263 and Some algebraic structures connected with the Yang-Baxter equation. Representations of quantum algebras, Funct. Anal. Appl. 17 (1983) 273.

[2] O. Foda, K. Iohara, M. Jimbo, R. Kedem, T. Miwa, H. Yan, An elliptic quantum algebra for $\widehat{s l}_{2}$, Lett. Math. Phys. 32 (1994) 259 and hep-th/9403094.

[3] R.J. Baxter, Exactly solved models in statistical mechanics, Academic Press, London, 1982.

[4] C. Frønsdal, Quasi Hopf Deformations of Quantum Groups, Lett. Math. Phys. 40 (1997) 117, q-alg/9611028.

[5] M. Jimbo, H. Konno, S. Odake, J. Shiraishi, Quasi-Hopf twistors for elliptic quantum groups, to appear in "Transformation Groups" and q-alg/9712029.

[6] S. Khoroshkin, D. Lebedev, S. Pakuliak, Elliptic algebra $A_{q, p}\left(\hat{s}_{2}\right)$ in the scaling limit, Commun. Math. Phys. 190 (1998) 597 and q-alg/9702002.

[7] M. Jimbo, H. Konno, T. Miwa, Massless XXZ model and degeneration of the elliptic algebra $\mathcal{A}_{q, p}(\widehat{s l}(2))$, in Ascona 1996, "Deformation theory and symplectic geometry", 117-138 and hep-th/9610079.

[8] H. Konno, Degeneration of the elliptic algebra $\mathcal{A}_{q, p}(\widehat{s l}(2))$ and form factors in the Sine-Gordon theory, Proceedings of the Nankai-CRM joint meeting on "Extended and Quantum Algebras and their Applications to Physics", Tianjin, China, 1996, to appear in the CRM series in mathematical physics, Springer Verlag, and hep-th/9701034. 
[9] S.M. Khoroshkin, V.N. Tolstoy, Yangian double and rational R matrix, Lett. Math. Phys. (1995) and hep-th/9406194.

[10] S.M. Khoroshkin, Central extension of the Yangian double, Collection SMF, 7ème rencontre du contact franco-belge en algèbre, Reims 1995, q-alg/9602031.

[11] K. Iohara, M. Kohno, A central extension of $\mathcal{D} Y_{\hbar}(\widehat{g l}(2))$ and its vertex representations, Lett. Math. Phys. 37 (1996) 319 and q-alg/9603032.

[12] K. Iohara, Bosonic representations of Yangian double $\mathcal{D} Y_{\hbar}(\widehat{g l})$ with $\widehat{g l}=\widehat{g l}_{N}, \widehat{s l}_{N}$, J. Phys. A (Math. Gen.) 29 (1996) 4593 and q-alg/9603033.

[13] S. Khoroshkin, A. LeClair, S. Pakuliak, Angular quantization of the Sine-Gordon model at the free fermion point, hep-th/9904082.

[14] N.Yu. Reshetikhin, M.A. Semenov-Tian-Shansky, Central extensions of quantum current groups, Lett. Math. Phys. 19 (1990) 133.

[15] E. Frenkel, N. Reshetikhin, Quantum affine algebras and deformations of the Virasoro and $W$-algebras, Commun. Math. Phys. 178 (1996) 237 and q-alg/9505025.

[16] H. Awata, H. Kubo, S. Odake, J. Shiraishi, Quantum $\mathcal{W}_{N}$ algebras and Macdonald polynomials, Commun. Math. Phys. 179 (1996) 401 and q-alg/9508011 and J. Shiraishi, H. Kubo, H. Awata, S. Odake, A Quantum Deformation of the Virasoro Algebra and the Macdonald Symmetric Functions, Lett. Math. Phys. 38 (1996) 33 and q-alg/9507034.

[17] B. Feigin, E. Frenkel, Quantum $W$ algebras and elliptic algebras, Commun. Math. Phys. 178 (1996) 653 and q-alg/9508009.

[18] J. Avan, L. Frappat, M. Rossi, P. Sorba, Poisson structures on the center of the elliptic algebra $\mathcal{A}_{q, p}\left(\widehat{s l}(2)_{c}\right)$, Phys. Lett. A 235 (1997) 323 and q-alg/9705012.

[19] J. Avan, L. Frappat, M. Rossi, P. Sorba, New $\mathcal{W}_{q, p}(\operatorname{sl}(2))$ algebras from the elliptic algebra $\mathcal{A}_{q, p}\left(\widehat{s l}(2)_{c}\right)$, Phys. Lett. A 239 (1998) 27 and q-alg/9706013.

[20] J. Avan, L. Frappat, M. Rossi, P. Sorba, Deformed $\mathcal{W}_{N}$ algebras from elliptic sl $(N)$ algebras, Commun. Math. Phys. 199 (1999) 697 and math.QA/9801105.

[21] J. Avan, L. Frappat, M. Rossi, P. Sorba, Universal contruction of $q$-deformed $\mathcal{W}$ algebras, to appear in Commun. Math. Phys. (1999), math.QA/9807048.

[22] A.A. Belavin, Dynamical symmetry of integrable quantum systems, Nucl. Phys. B 180 (1981) 189.

[23] D.V. Chudnovsky, G.V. Chudnovsky, Completely X-symmetric S-matrices corresponding to theta functions, Phys. Lett. A 81 (1981) 105.

[24] M. Jimbo, T. Miwa, Quantum KZ equation with $|q|=1$ and correlation functions of the XXZ model in the gapless regime, J. Phys. A (Math. Gen.) 29 (1996) 2923 and hep-th/9601135.

[25] A.P. Prudnikov, Yu.A. Brychkov, O.I. Marichev, Integrals and Series, Gordon and Breach Sciences Publishers, 1986.

[26] D. Arnaudon, J. Avan, L. Frappat, É. Ragoucy, M. Rossi and P. Sorba, in preparation. 